Historic, Archive Document

Do not assume content reflects current scientific knowledge, policies, or practices. 



\title{
PRICE-LIST of PEONIES and IRISES
}

\author{
Grown by E. M. Buechly, Greenville, Ohio
}

We feel very grateful for the liberal patronage given us in the past, and hope to merit your favors in the future. Many of our friends may have our descriptive catalogue of last season on file, therefore we have decided to issue only a brief price-list at this time and those who do not have the descriptive catalogue will be supplied on request. And later, we hope to issue a new catalogue to be placed on file for reference. With this end in view we ask each one who receives self-addressed card to kindly fill with the information desired in order to revise our mailing list that it contain names of interested parties only.

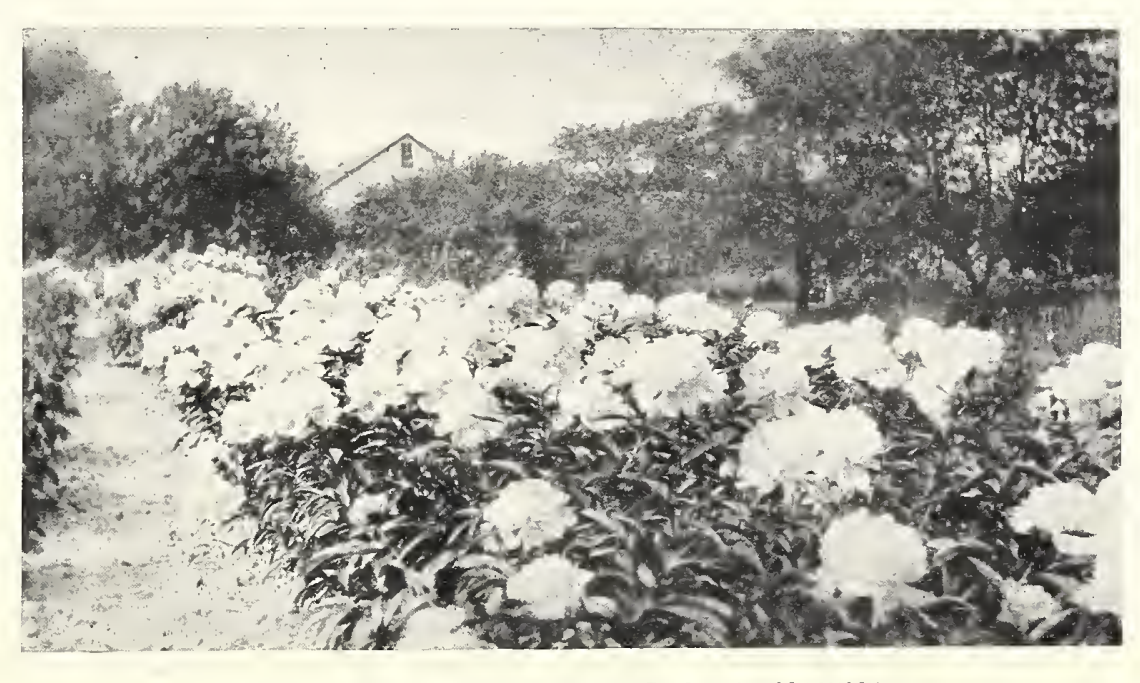

A ROW OF PRIZE WINNING FESTIVA MAXIMA

standard divisions of from three to five eyes, in most cases from dividing two to three year old plants. We do not recommend the so-called one eye divisions, but to meet a demand for such, we will supply
of any peonies selling for $\$ 3.00$ or more each, at one-half the list price for standard divisions.

Orders should be sent as early as possible, as stock of rarest varieties is limited, and shipments are made in the order received. Please advise whether shipments are to be made by express or parcel post.

\section{GUARANTEE}

We guarantee safe arrival of plants, and that they are Prices listed herein cancel all previous quotations.

Size of plants, at the prices quoted, are for good, strong

\section{PEONIES}

Grade

clibert crouste

lrebie Brimd

Alyustin d'Hollr

Iralancle

. Baroness selorveder

5 Beante Francaise

0 Berlioz

4 Chestine Gow

Claire bubois.

1 Courome Io'0

borehester

Dr. Boistuval

Dnchess de Nemouns

Edulis silperba

6 Ella Wheeler Wilcox .. $1.50 \quad 7.9$

.2 Elizabeth Barlet Browning 7.5

8.7 Elwoud pleas . . . . 20.007 .9

8.7 Enchantrest

8.0 Etta

3 Eugene Verdie

Engenie Verdie

Felix Cromsise

Festiva

Festiva Maxinla

Florence Nightingale

Frances c. iriỏiard.

Gallatliee

Georgiana Shaylor

Germaine Bigot ......

Ginette $\ldots \ldots \ldots \ldots \ldots . .$.

9 Cloire de Chas. Gombanlt

(4............... 1.00

Governor Morton ..... 5.00

Grace Loomis ......... 25.00 8

8.8 Franditlora

Grover Clevelaud . . 2.008 .6

Hamiett Farmsley ....

Henri Murger

James Kelway

Jeanne d'Are

Jubilee
8.6 Judge Berry
8.8 Kinl Rosentieid

1.009 .8

$\begin{array}{ll}3.00 & 9.1 \\ 1.50 & 8.8\end{array}$

$1.00 \quad 7.8$

$1.25 \quad 7.9$

$\begin{array}{ll}1.00 & 8.5 \\ 1.00 & 8.4\end{array}$

$5.00 \quad 9.9$

759.

$.75 \quad 6.9$

1.008 .8

$\begin{array}{ll}6.00 & 8.5 \\ 6.00 & 8.9\end{array}$

$2.00 \quad 7.7$

$1.50 \quad 7.9$

1.009 .4

.75
.75

.758 .1

$2.00 \quad 8.3$

$5.00 \quad 8.9$

.008 .5

.00 7.9

$\begin{array}{ll}9.1 \\ 8.00 & 7.5\end{array}$

8.4

9.4

$1.00 \quad 7.7$

1.509

$1.50 \quad 8.3$

$2.00 \quad 9.2$

$9.00 \quad 7.8$

Lady Beresfold

aldy Iris

La Perle

La Fiancee

Le cygne

Livingstome

Loveliness

Mireille

Mikado

Milton Hili healthy and true to name, and hold ourselves in readiness to replace two for one any that prove otherwise.

Kelway's glorious

8.008 .4 Ilont Blane

3.007 .8 MIs. Carew

Felivays (x)

$30.60 \quad 9.9$

Lady Ilexander I)

15.008

Laura Dessert ....... 15.00

Louis Van Houtte

Maliel I. I'ranklin.

2.50

6.00

1.25
2.00
18.00

Madamo Boulauger ....

Madane De Vernerille.

Madame De Galliau..

Vadane Emile Galle

10.009

1.50
.75

Madame Emile Galle .. 1.00

Hadame Forel ........ 1.00

Madante Geisler ...... 1.00

Madame, Jules Dessert 6.00

$\ldots \ldots \ldots \ldots \ldots \ldots \ldots$...........

Mademoiselle Roussean 1.00

Marie Stnart ...... $75 \quad 9.4$

Marie Jaequin ....... 1.00

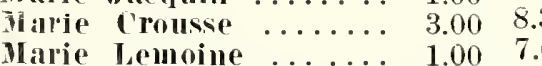

Matlilde de Roseneck.. 1.00

Martlia Bulloelı ...... 20.00

Mary Baker Eddy ..... 1.00

Marguerite Gerard .... 1.25

Mary Woodhury shay lor 35.00

Midsumuer Night's Dream

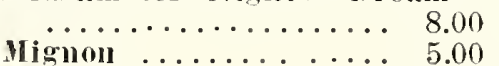

Monseur Dupout

Monseur Tules Elie ....

Iodel de Perfection...
7.50

$\begin{array}{ll}8.00 & 8.7 \\ 1.50 & 9.7\end{array}$

$\begin{array}{rr}.75 & 8.7 \\ 4.50 & 8.8\end{array}$

$30.00 \quad 9$

.75
.75
1.00

1.009

Irs. Edward Harding.

Mrs. Geo. IBumatrol.

Getarie Demly

Otificiualis Rubri

opal

ricetion

Plitlip Rivoire

Phoebe cary

President Tait

President Wilson

Raonl Dessert

Reine Hortense

Rieluard Carvel

Rosilara Superba

Rutli Brand

Samali Beruhadid

Solange

Some Ganako

snow Wlieel

8.3 Splendida

7.4 Solv. de Gaspard Calot

Suzanme bessert

Siteplianie

'Throrese

Tourangelle

Inlorlat

Viluinie

3 Walter Faxom

\section{IRISES}

Grade

8.9. Ileurar

each

1.00

Ambassadeur ...4 4.50

1.008 .4 Allla Farl ...... 2.00

3.008 .3 Anmie Leslie ... 2.00

3.008 .1 Archereque

1.007 .1 Blue Jay

1.00 Blue King (Orientalis)

4.00

1.00 lletla

$\begin{array}{rr}5.00 & 6.9 \\ 1.00 & 8 . \\ 50.00 & \\ 4.00 & 7 . \\ 1.00 & 8 . \\ .75 & 6.5 \\ 6.00 & 7.9 \\ 1.50 & 8.1 \\ 25.00 & 7.8 \\ 10.00 & 9 . \\ 1.00 & 8 \\ 2.00 & 9 \\ 50.00 & 7 . \\ 15.00 & 7.4 \\ 2.00 & 9 \\ 6.00 & \\ 6.00 & 8 \\ .75 & 7 \\ 2.00 & 8 \\ 3.50 & 7.8 \\ 8.00 & 8.8 \\ .50 & \\ 4.00 & 7.7 \\ 2.00 & 8.3 \\ 5.00 & 8.4 \\ 1.00 & 7.8 \\ 1.50 & 8.4 \\ 3.50 & 8.1 \\ 5.00 & 7.6 \\ 5.00 & \\ .75 & \\ 1.00 & 7 \\ 1.00 & 8.3 \\ 7.50 & \\ \end{array}$

Cliester Hunt

Fluard Micluell .00

Glory of Reading. 40

HeV Majesty .... .35

Isolene $\ldots \ldots \ldots \ldots . .50$

Iriquois $\ldots \ldots \ldots \ldots . \quad .35$

Juniata ........

Kóliii

3.00
3.00

2.50

2.00

2.00

2.00

lord of Jine ... 2.00

Lorely

Mme. Cherean ... .20

Magnitiea $\ldots \ldots . .5 .00$

lliss Eardley .... .50

Monsignior ..... .40

Narajo ........ .50

Opera ......... 3.50

Oriflamme ..... .75

Pallida Dalmatica .30

Pauline ........ .50

Pocahontas ......

Prosper Langier. $40-2.50$

Quaker Lady ... $.50 \quad 3.50$

Red Cloud ..... .50

Rliein Nixie

Sherbert

Sherwin Wriglit.. .40

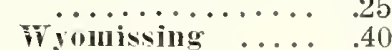

8.3 White Knight $\ldots 1.00$

General list of Gladiolii will be issued later.

Jerennial Jelphineum, or Englislı Larkspul, these are in beautiful shades of blue, from a mere tint thru all shades to dark blue and purple; 5 to $6 \mathrm{ft}$. high second year. Beautiful. Plants per doz.

$\$ 7.50$

3.00

2.00 Seed per large packä..... . .25

Address all communications to

E M. BUECHLY Greenville, Ohio
Snow Queen (Orientalis) 
<smiles>[CH-]=C</smiles> 